\title{
Tinea Capitis in Adults; A 18-year Epidemiological, Clinical and Mycological Study in Korea
}

\section{Jin Park' ${ }^{1,2}$, Su-Kyung Park1, Seok-Kweon Yun ${ }^{1,2}$, Han-Uk Kim ${ }^{1,2}$}

${ }^{1}$ Department of Dermatology, Chonbuk National University Medical School, Jeonju, Korea, ${ }^{2}$ Research Institute of Clinical Medicine of Chonbuk National University-Biomedical Research Institute of Chonbuk National University Hospital, Jeonju, Korea

Background: Tinea capitis (TC), a dermatophyte infection involving hair and scalp, occurs primarily in prepubertal children. The information of this disease entity in adults are very scarce.

Objectives: We aimed to comprehensively determine the current epidemiological, clinical and mycological characteristics of adult TC in Korea.

Materials and methods: We retrospectively evaluated 82 adults (44.3\%) among the 185 TC patients from a tertiary hospital between June 2000 and June 2017.

Results: Mean age of patients was $66.9 \pm 15.8$ years $(20-90$ years) with female predominance (male: female ratio 1:3.1). Mean duration of disease onset until the mycological diagnosis was 22.5 weeks (1-144 weeks) and the misdiagnosed rate through clinical examination was $65.9 \%$. The most common presumptive initial diagnosis was seborrheic dermatitis $(n=20$, $24.4 \%$ ), followed by bacterial folliculitis ( $n=15,18.3 \%$ ), allergic contact dermatitis $(n=5,6.1 \%)$, psoriasis $(n=4,4.9 \%)$, cellulitis $(n=3,3.7 \%)$, unclassified eczema $(n=3,3.7 \%)$, telogen effluvium $(n=2,2.4 \%)$, lupus erythematosus $(n=1,1.2 \%)$, and acne $(n=1$, $1.2 \%)$. Underlying chronic systemic illness were found in 61 patients (74.4\%); hypertension (22.0\%), diabetes (14.6\%), malignancy (12.2\%), liver/gastro-intestinal disease (8.5\%), heart disease $(7.3 \%)$, kidney disease $(6.1 \%)$, thyroid disease $(4.9 \%)$, and pulmonary disease (3.7\%). On clinical features, pustular type was shown in $26.8 \%$ patients, followed by seborrheic dermatitis-like (25.6\%), gray patch (23.2\%), kerion celsi $(22.0 \%)$, and black dot (2.4\%) type. Accompanying alopecia was shown in 46 patients $(56.1 \%)$

Table 1. Sex and age distribution of tinea capitis patients

\begin{tabular}{|c|c|c|c|}
\hline \multirow{2}{*}{$\begin{array}{c}\text { Age } \\
\text { (years) }\end{array}$} & \multicolumn{2}{|c|}{ Sex } & \multirow{2}{*}{ Total (\%) } \\
\hline & Male & Female & \\
\hline $0 \sim 9$ & 45 & 44 & $89(48.1)$ \\
\hline $10 \sim 19$ & 5 & 9 & $14(7.6)$ \\
\hline 20 29 & 3 & 0 & $3(1.6)$ \\
\hline 30 39 & 2 & 2 & $4(2.2)$ \\
\hline $40 \sim 49$ & 2 & 1 & $3(1.6)$ \\
\hline $50 \sim 59$ & 3 & 5 & $8(4.3)$ \\
\hline $60 \sim 69$ & 3 & 20 & $23(12.4)$ \\
\hline $70 \sim$ & 7 & 34 & $41(22.2)$ \\
\hline Total & 70 & 115 & $185(100.0)$ \\
\hline
\end{tabular}

Figure 1. Variable clinical presentation in adult tinea capitis. (A) Pustular type with diffuse hair loss, (B) Seborrheic dermatitis-like type with no apparent hair loss, (C) Gray patch type showing localized bald patch, (D) Kerion celsi,

(E) Black dot type

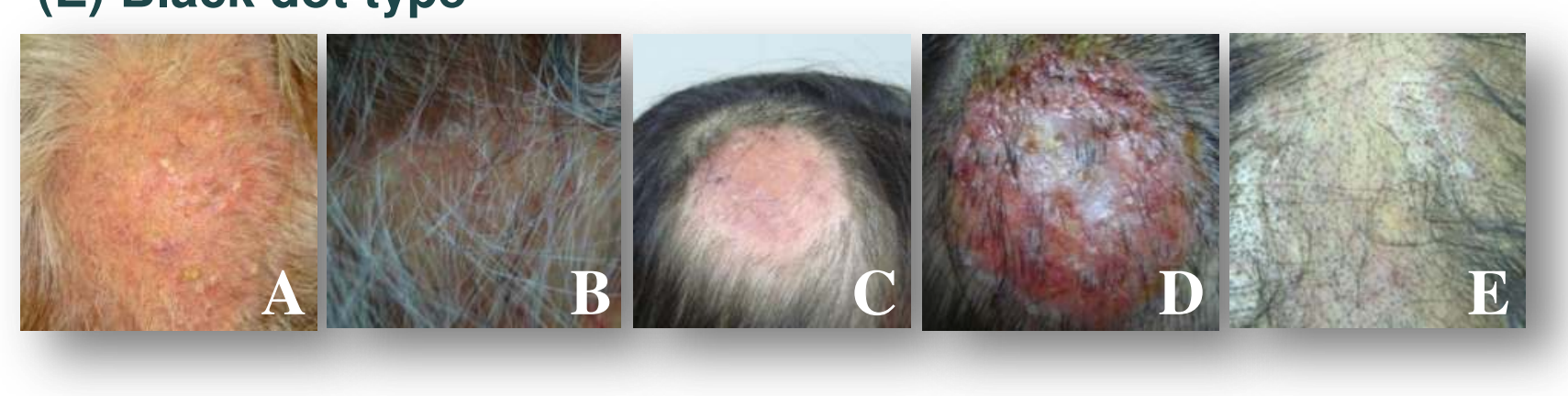

Forty eight patients (58.5\%) also had the tinea infection at other skin area; tinea faciale $(n=32,39.0 \%)$, tinea corporis $(n=8$, $9.8 \%)$, tinea pedis $(n=5,6.1 \%)$, and onychomycosis $(n=3,3.7 \%)$. Among 23 culture-positive patients, M. canis $(n=13,56.5 \%)$ was the most common causative organism, followed by $T$. rubrum $(\mathrm{n}=5,21.7 \%), \quad T$. mentagrophytes $(\mathrm{n}=4,17.4 \%)$, and $T$. verrucosum $(n=1,4.4 \%)$. Most patients (92.7\%) achieved a complete resolution. The duration of the treatment was 1 week to 27 weeks. Six patients were lost of follow-up period. Three patients $(3.7 \%)$ healed with permanent alopecia and 7 patients $(9.2 \%)$ demonstrated a recurrence.

\section{Conclusion}

We report the largest case series of TC in adults, and most recent survey of TC in Korea. The prevalence of adult TC in this study is significantly higher than those of earlier studies in Koreans was well as other countries. TC of adult occurs predominantly on postmenopausal women ( $\geq 60$ years) and frequently accompanies chronic medical illness or immunosuppression. Adult TC shows distinctive clinical and mycological features differentiating from prepubertal TC, and it may lead to wrong or delay in diagnosis. Regarding on the causative organism, the prevalence of anthrophophilic species, such as $T$. rubrum is relatively higher than those of children.

Table 2. Summary of Tinea capitis in adult in the literatures

\begin{tabular}{|c|c|c|c|c|c|c|c|}
\hline $\begin{array}{l}\text { Authors } \\
\text { (years, } \\
\text { country) }\end{array}$ & $\begin{array}{c}\text { Case } \\
\text { number } \\
\text { (propor } \\
\text { tion of } \\
\text { adult) }\end{array}$ & $\begin{array}{c}\text { Age } \\
\text { Range } \\
\text { (Mean) }\end{array}$ & $\begin{array}{l}\text { Sex } \\
(M: F)\end{array}$ & $\begin{array}{c}\text { Diseas } \\
\text { e } \\
\text { duratio } \\
\mathbf{n}\end{array}$ & $\begin{array}{l}\text { Other } \\
\text { site } \\
\text { involve } \\
\text { ment }\end{array}$ & $\begin{array}{l}\text { Underlyin } \\
\text { g chronic } \\
\text { disease }\end{array}$ & $\begin{array}{l}\text { Isolated species } \\
\text { (order of frequency) }\end{array}$ \\
\hline $\begin{array}{l}\text { Oh et al. } \\
(1978-1988, \\
\text { Korea })\end{array}$ & $\begin{array}{c}41 \\
(2.0 \%)\end{array}$ & $\begin{array}{l}21-85 \\
(58.5)\end{array}$ & $1: 19.5$ & $N / A$ & $14.6 \%$ & $\mathrm{~N} / \mathrm{A}$ & $\begin{array}{l}\text { M.canis > T.ferrugineum, } \\
\text { T.mentagrophyte, } \\
\text { T.rubrum, T.verrucosum }\end{array}$ \\
\hline $\begin{array}{l}\text { Aste et al. } \\
\text { (1973-1994, } \\
\text { Italy) }\end{array}$ & $\begin{array}{c}17 \\
(\mathrm{~N} / \mathrm{A})\end{array}$ & $\begin{array}{l}17-76 \\
(\mathrm{~N} / \mathrm{A})\end{array}$ & $\begin{array}{c}\text { All } \\
\text { female }\end{array}$ & $\begin{array}{c}8-10 \\
\text { months }\end{array}$ & $\mathrm{N} / \mathrm{A}$ & $29.4 \%$ & $\begin{array}{l}\text { M.canis > T.violaceum, } \\
\text { T.mentagrophyte } \\
>\text { T.verrucosum }\end{array}$ \\
\hline Devliotou- & & & & & & & T.violaceum > M.canis > \\
\hline $\begin{array}{l}\text { Panagliotidou } \\
\text { et al. } \\
\text { (1981-95, } \\
\text { Greece) }\end{array}$ & $\begin{array}{c}35 \\
(5.8 \%)\end{array}$ & $\begin{array}{r}19-89 \\
(\mathrm{~N} / \mathrm{A})\end{array}$ & $1: 16.5$ & $\begin{array}{c}1 m-30 \\
\text { years }\end{array}$ & $28.6 \%$ & $20.0 \%$ & $\begin{array}{l}\text { T.rubrum, T.verrucosum } \\
\text { > T.schoenleinii, } \\
\text { T.mentagrophyte > } \\
\text { T.tonsurans }\end{array}$ \\
\hline $\begin{array}{l}\text { El-Khalawany } \\
\text { et al. (2002- } \\
\text { 2012, Egypt) }\end{array}$ & $\begin{array}{c}58 \\
(4.2 \%)\end{array}$ & $42.9 \pm 8.54$ & $1: 5.4$ & $\begin{array}{c}7.1 \pm 2.4 \\
1 \\
\text { months }\end{array}$ & $29.3 \%$ & $34.4 \%$ & $\begin{array}{l}\text { T.violaceum > } \\
\text { M.audouinii > M.canis } \\
>\text { T.schoenleini }\end{array}$ \\
\hline $\begin{array}{c}\text { Mebazza A et } \\
\text { al } \\
(1990-2005 \\
\text { Tunisia }\end{array}$ & $\begin{array}{c}60 \\
(5.3 \%)\end{array}$ & $\begin{array}{l}18-75 \\
(34.5)\end{array}$ & $1: 2.3$ & $\begin{array}{c}1-52 \\
\text { weeks }\end{array}$ & $50 \%$ & $\mathrm{~N} / \mathrm{A}$ & $\begin{array}{l}\text { T.violaceum }>\text { M.canis }> \\
T \text {. schoenleini }>T . \\
\text { verrucosum }>T \text {. } \\
\text { mentagrophytes }\end{array}$ \\
\hline $\begin{array}{l}\text { Cervetti et al. } \\
\text { (1997-2012, } \\
\text { Italy) }\end{array}$ & $\begin{array}{c}13 \\
(2.6 \%)\end{array}$ & $\begin{array}{c}29-80 \\
(56.5)\end{array}$ & 1:2.2 & $\mathrm{N} / \mathrm{A}$ & $38.5 \%$ & $23.1 \%$ & $\begin{array}{l}\text { M.canis > } \\
\text { T.mentagrophyte > } \\
\text { T.rubrum } \\
>\text { T.violaceum }\end{array}$ \\
\hline $\begin{array}{l}\text { Present data } \\
\text { (2000-2017, } \\
\text { Korea) }\end{array}$ & $\begin{array}{c}82 \\
(44.3 \%)\end{array}$ & $\begin{array}{c}20-90 \\
(66.9 \pm \\
15.8)\end{array}$ & 1:3.1 & $\begin{array}{c}22.5 \\
\text { weeks }\end{array}$ & $46.2 \%$ & $74.4 \%$ & $\begin{array}{l}\text { M.canis > T.rubrum > } \\
\text { T.mentagrophyte, } \\
\text { T.verrucosum }\end{array}$ \\
\hline
\end{tabular}

References

Oh SH, Kim SH, Suh SB. Tinea capitis of adults in Taegu city for 11 years (1978 1988). Korean J Dermatol 1989;27:666-679.

Aste N, Pau M, Biggio P. Tinea capitis in adults. Mycoses 1996;39:299-301. Devliotou-Panagliotidou D, Koussidou-Eremondi T, Chaidemenos GC, Theodoridou M, Minas A. Tinea capitis in adults during 1981-95 in northern Greece. Mycoses 2001:44:398-400.

El-Khalawany M, Shaaban D, Hassan H, Abdalsalam F, Eassa B, Abdel Kader A, Shaheen I. A multicenter clinicomycological study evaluating the spectrum of adult tinea capitis in Egypt. Acta Dermatovenerol Alp Pannonica Adriat. 2013;22:77-82.

Mebazaa A, EL Oumari K, Ben Said M, Ghariani N, Denguezli M, Mili AF, et, al. Tinea capitis in adults in Tunisia. Int J dermatol 2010;49:513-516.

Cervetti O, Albini P, Arese V, Ibba F, Novarino M, Panzone M. Tinea capitis in adults. Advances in Microbiology 2014;4:12. 